\title{
Weyl Anomaly Induced Current in Boundary Quantum Field Theories
}

\author{
Chong-Sun $\mathrm{Chu}^{1,2}$ and Rong-Xin Miao ${ }^{1,3}$ \\ ${ }^{1}$ National Center for Theoretical Sciences, National Tsing-Hua University, Hsinchu 30013, Taiwan \\ ${ }^{2}$ Department of Physics, National Tsing-Hua University, Hsinchu 30013, Taiwan \\ ${ }^{3}$ School of Physics and Astronomy, Sun Yat-Sen University, Zhuhai 519082, China
}

(Received 20 March 2018; revised manuscript received 12 November 2018; published 18 December 2018)

\begin{abstract}
We show that when an external magnetic field parallel to the boundary is applied, the Weyl anomaly gives rises to a new anomalous current in the vicinity of the boundary. The induced current is a magnetization current in origin: the movement of the virtual charges near the boundary give rise to a nonuniform magnetization of the vacuum and hence a magnetization current. Unlike other previously studied anomalous current phenomena such as the chiral magnetic effect or the chiral vortical effect, this induced current does not rely on the presence of a material system and can occur in vacuum. Similar to the Casimir effect, our discovered phenomenon arises from the effect of the boundary on the quantum fluctuations of the vacuum. However this induced current is purely quantum mechanical and has no classical limit. We briefly comment on how this induced current may be observed experimentally.
\end{abstract}

DOI: 10.1103/PhysRevLett.121.251602

Introduction.-The quantum transportation of charges induced by a quantum anomaly induced current is an interesting phenomenon. Much has been discussed in the literature [1]. A number of such effects are known. The famous one is the chiral magnetic effect (CME) [2-6], which refers to the generation of currents parallel to an external magnetic field $\boldsymbol{B}$. The chiral vortical effect (CVE) [7-10] refers to the generation of a current due to rotational motion in the charged fluid. The induced currents take the forms

$\boldsymbol{J}_{V}=\sigma_{(\mathcal{B}) V} \boldsymbol{B}+\sigma_{(\mathcal{V}) V} \boldsymbol{\omega}, \quad \boldsymbol{J}_{A}=\sigma_{(\mathcal{B}) A} \boldsymbol{B}+\sigma_{(\mathcal{V}) A} \boldsymbol{\omega}$,

where $\sigma_{(\mathcal{B}) V}=\left(e \mu_{A} / 2 \pi^{2}\right), \sigma_{(\mathcal{B}) A}=\left(e \mu_{V} / 2 \pi^{2}\right)$ are the chiral magnetic conductivities, $\sigma_{(\mathcal{V}) V}=\left(\mu_{V} \mu_{A} / \pi^{2}\right), \sigma_{(\mathcal{V}) A}=$ $\left(\mu_{V}^{2}+\mu_{A}^{2} / 2 \pi^{2}\right)+\left(T^{2} / 6\right)$ are the chiral vortical conductivities, $\mu_{A}, \mu_{V}$ are the chemical potentials, and $T$ is the temperature of the medium. The chemical potential dependent induced current arises as a result of an imbalance in the left and right moving modes due to the axial anomaly, while the temperature dependent part comes from the gravitational anomaly [11]. More recently, it has also been pointed out that an anomalous current also occurs in a conformally flat gravitational spacetime due to the Weyl anomaly $[12,13]$. It should be noted that these anomalous currents occur only in a material system where the chemical

Published by the American Physical Society under the terms of the Creative Commons Attribution 4.0 International license. Further distribution of this work must maintain attribution to the author(s) and the published article's title, journal citation, and DOI. Funded by SCOAP ${ }^{3}$. potentials are nonvanishing, or in a curved spacetime. Since the axial anomaly is an intrinsic property of quantum field theory (QFT), which is present even in a flat spacetime and in a vacuum, it is natural to ask whether the phenomenon of anomalous current may also occur in a flat spacetime due to the quantum fluctuation of the vacuum.

The Casimir effect is one of the most well-known manifestations of the quantum fluctuation of an electromagnetic vacuum in the presence of a boundary [14-16]. Recently the Casimir effect has been analyzed in full generality for an arbitrary shape of boundary and for an arbitrary spacetime metric, and new universal relations between the Casimir coefficients and the boundary central charge in a boundary conformal field theory have been discovered [17]. The presence of a boundary has also many other interesting physical consequences, e.g., renormalization group flows and critical phenomena [18], or the topological insulator [19], etc.

In this Letter, we show that for a general class of boundary quantum field theory (BQFT) with $U(1)$ gauge symmetry, the quantum Weyl anomaly of the theory induces a new kind of induced current near the boundary. Consider a general BQFT defined on a four-dimensional spacetime manifold $M$ with coordinates $x^{\mu}$, and has a boundary $\partial M$ with coordinates $y^{a}$. The Weyl anomaly can be defined as the difference between the trace of renormalized stress tensor and the renormalized trace of stress tensor $[20,21]$. We find it useful to introduce the following integrated Weyl anomaly

$$
\mathcal{A}=\int_{M} \sqrt{g}\left[g^{\mu \nu}\left\langle T_{\mu \nu}\right\rangle-\left\langle g^{\mu \nu} T_{\mu \nu}\right\rangle\right]
$$


$\mathcal{A}$ is equal to the variation of the effective action with respect to constantly rescaling the metric [22]. For simplicity, we focus on QFT, which are covariant, gauge invariant, unitary, and renormalizable, e.g., QED. By "renormalizable," we mean, in the sense of perturbation theory, that all the coupling constants are of non-negative mass dimension. We also assume that the Weyl anomaly depends on only the positive powers of the coupling constants (including the mass $m$ ), so that it has a welldefined limit when we turn off the coupling constants. For this class of QFT, $\mathcal{A}$ takes the following form [20,23]:

$\mathcal{A}=\int_{M} \sqrt{g}\left[b_{1} F_{\mu \nu} F^{\mu \nu}+O\left(R^{2}\right)\right]+\int_{\partial M} \sqrt{h} O(R k)$.

Here $O\left(R^{2}\right)$ denotes terms constructed out of the bulk curvature tensor, including terms with positive powers of coupling constants; e.g., $R^{2}, \quad R_{\mu \nu} R^{\mu \nu}, R_{\mu \nu \alpha \beta} R^{\mu \nu \alpha \beta}$, $\square R, m^{2} R, m^{4}, \cdots$, and $O(R k)$ denote the boundary Weyl anomaly $[28,29]$ that is constructed out of the boundary curvature tensor and the exterior curvature of the boundary. $b_{1}$ is the bulk central charge which govern the gauge field contribution to the Weyl anomaly [Eq. (3)]. For the normalization of the gauge field kinetic term $S=-1 /\left(4 e^{2}\right) \int F^{2}, b_{1}$ is related to the beta function as $b_{1}=-[\beta(e)] /\left(2 e^{3}\right)[30]$. Below we show that for general $\mathrm{BQFT}$, as specified above, the expectation value of the induced current at a distance $x$, which is very close to the boundary [31], is given by

$$
\langle\boldsymbol{J}\rangle=\frac{e^{2} c}{\hbar} \frac{4 b_{1} \boldsymbol{n} \times \boldsymbol{B}}{x}, \quad x \sim 0,
$$

where $\boldsymbol{n}$ is the inner normal to the boundary. The current [Eq. (4)] is a magnetization current $\boldsymbol{J}=\nabla \times \boldsymbol{M}$, and it corresponds to a quantum magnetization

$$
\langle\boldsymbol{M}\rangle=\frac{e^{2} c}{\hbar} 4 b_{1} \log x \boldsymbol{B}
$$

of the vacuum. It is remarkable that the anomalous current [Eq. (4)] and the vacuum magnetization [Eq. (5)] take place even in a flat spacetime and at zero temperature. This is a pure quantum effect since it is inversely proportional the Planck constant and has no classical limit $\hbar \rightarrow 0$. The induced current is measured by a quantum Hall conductance $\sigma_{H}=e^{2} / \hbar$, which governs the quantum Hall effect. In fact the current [Eq. (4)] is in resemblance to the quantum Hall effect except that the current now is parallel to the boundary instead of perpendicular to the boundary as in the case of the standard Hall effect. One may therefore refer to Eq. (4) as an anomalous quantum Hall effect [32].

Physical Picture.-To understand the physical origin of the current [Eq. (4)] and the magnetization [Eq. (5)], let us consider for simplicity the set-up of a BQFT in a flat

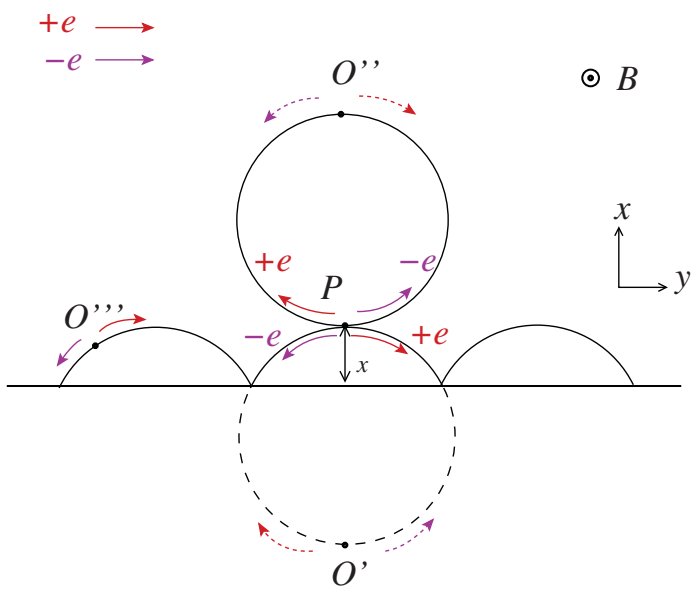

FIG. 1. Induced current from a virtual pair creation in the presence of a boundary.

spacetime with a flat boundary, see Fig. 1. Consider a point $P$ at the distance $x$ from the boundary. We are interested in the amount of charges passing through $P$ due to the vacuum process of a virtual particle creation and annihilation. Suppose that there is a magnetic field normal to (pointing out of) the figure, the charged particles will move along circles due to the Lorentz force. If there is no boundary, the virtual particle pairs created by quantum fluctuations at $O^{\prime}$ would annihilate at $P$ after moving along the dotted circle. This give rises to a transport of charges to the right. This is however precisely canceled by the movement of charges due to the quantum fluctuation at the point $O^{\prime \prime}$. Summing over all possible locations of the source points, it is clear that there is no net transport of charges induced at $P$. The situation is different when there is a boundary. In this case, those contributions from source points at $x<0$ are missing. This leads to a net amount of charges moving in the $-y$ direction. In addition, vacuum pairs created at source point $O^{\prime \prime \prime}$ could now reach $P$ due to the (virtual) reflection of the boundary. What exactly happens, perfect reflection or partial absorption, will depend on the boundary condition. But in any case there will be a net separation of charges and this contributes a transport of charges to the $+y$ direction. The current [Eq. (4)] can also be understood as a result of the magnetic response of the vacuum to the presence of a boundary. As we noted already, quantum fluctuation of the vacuum leads to temporary creation of virtual pair of charged particles, which are then guided to move in circles in the presence of a magnetic field. As a result, tiny current loops are formed with the positively and negatively charged virtual particles contributing in the same way to the magnetic dipole moment. Summing up all these contribution results in a total magnetization $\boldsymbol{M}$ of the vacuum. When there is no boundary, $\boldsymbol{M}$ is just an infinite constant that can be subtracted away by renormalization, and the renormalized vacuum magnetization $\langle\boldsymbol{M}\rangle=0$ has no physical effect. When there is a boundary, it is clear that 
the renormalized $\langle\boldsymbol{M}\rangle$ is zero distance from the boundary, but this becomes nontrivial near the boundary. This is very much like the Casimir effect. The magnetization [Eq. (5)] of the vacuum is a new effect and occurs only because of the presence of the boundary. Let us now turn to the rigorous QFT derivation.

Rigorous derivation.-We start with a proper analysis of the structure of the renormalized current $J^{\mu}$ near the boundary. In general, for a BQFT, the renormalized current is generally singular near the boundary and the expectation value takes the asymptotic form near $x \sim 0$ :

$\left\langle J_{\mu}\right\rangle=\frac{1}{x^{3}} J_{\mu}^{(3)}+\frac{1}{x^{2}} J_{\mu}^{(2)}+\frac{1}{x} J_{\mu}^{(1)}+\log x J^{(0)}+\cdots$,

where $\cdots$ denotes terms regular at $x=0$, and $J_{\mu}^{(n)}$ depends only the background geometry, the background vector field strength, and the type of fields under consideration. Hereafter we will drop the symbol \langle\rangle for the expectation value. A similar expansion has been considered for the renormalized stress tensor [33]. We consider current that is conserved $\left(D_{\mu} J^{\mu}=0\right)$ up to possibly an anomaly term. Since this term is finite, it is irrelevant to the divergent part of a renormalized current [Eq. (6)]. As a result, we obtain the gauge invariant solution

$$
\begin{aligned}
& J_{\mu}^{(3)}=0, \quad J_{\mu}^{(2)}=0, \\
& J_{\mu}^{(1)}=\alpha_{1} F_{\mu \nu} n^{\nu}+\alpha_{2} \mathcal{D}_{\mu} k+\alpha_{3} \mathcal{D}_{\nu} k_{\mu}^{\nu}+\alpha_{4} \star F_{\mu \nu} n^{\nu}
\end{aligned}
$$

where $F_{\mu \nu}, \star F_{\mu \nu}, n_{\mu}, \mathcal{D}_{m}, k_{\mu \nu}$, and $h_{\mu \nu}$ are, respectively, the background field strength, Hodge dual of field strength, the normal vector, the induced covariant derivative, the extrinsic curvature, and the induced metric of the boundary. Here the coefficients $\alpha_{i}$ are arbitrary and the expression [Eq. (7)] gives the most general form of boundary behavior of the current that is consistent with the conservation law and gauge invariance. We will now show that these current coefficients are indeed completely fixed by the central charges of the theory.

To establish this result, let us follow an observation of Ref. [17] that allows one to relate the variation of $\mathcal{A}$ with the asymptotic form of the stress tensor near the boundary. For the present case of a current, we have the relation

$$
(\delta \mathcal{A})_{\partial M_{\epsilon}}=\left(\int_{M} \sqrt{g} J^{\mu} \delta A_{\mu}\right)_{\log \frac{1}{\epsilon}},
$$

where a regulator $x \geq \epsilon$ to the boundary is introduced for the integral on the right hand side (RHS) of Eq. (8). The relation [Eq. (8)] identifies the boundary contribution of the variation of the integrated anomaly $\mathcal{A}$ under an arbitrary variation of the gauge field $\delta A_{\mu}$ with the UV logarithmic divergent part of the integral involving the expectation value $J^{\mu}$ of the renormalized $U(1)$ current. The power of the relation [Eq. (8)] lies in the fact that the left hand side of Eq. (8) is a total variation and imposes constraints on the RHS of Eq. (8) that are powerful enough to fix completely the asymptotic behavior of the current in terms of the Weyl anomaly of the theory. We refer the readers to the Supplemental Material [34] for the derivation of this key relation [Eq. (8)].

Now let us use (8) to fix the current coefficients. To proceed, let us consider the metric written in the Gauss normal coordinates $d s^{2}=d x^{2}+\left(h_{a b}-2 x k_{a b}+\cdots\right) d y^{a} d y^{b}$, where $x \in[0,+\infty)$, and $n_{\mu}=(1,0,0,0)$ is the inward pointing normal vector. We also choose a gauge $A_{x}=0$ and expand the gauge field about the boundary: $A_{b}=$ $a_{b}+x A_{b}^{(1)}+\cdots$. Taking the variation of the Weyl anomaly [Eq. (3)] with respect to the gauge field, we have $(\delta \mathcal{A})_{\partial M}=4 b_{1} \int_{\partial M} \sqrt{h} F^{b}{ }_{n} \delta a_{b}$. Next, when we substitute Eq. (6) and Eq. (7) into the RHS of Eq. (8), integrate over $x$, and select the logarithmic divergent term, we obtain $\left(\int_{M} \sqrt{g} J^{\mu} \delta A_{\mu}\right)_{\log 1 / \epsilon}=\int_{\partial M} \sqrt{h}\left(\alpha_{1} F^{b}{ }_{n}+\alpha_{2} \mathcal{D}^{b} k+\alpha_{3} \mathcal{D}_{j} k^{j b}+\right.$ $\left.\alpha_{4} \star F^{b}{ }_{n}\right) \delta a_{b}$. As a result, we obtain, for a unitary QFT without the parity odd anomaly term [23], $\alpha_{1}=4 b_{1}$, $\alpha_{2}=\alpha_{3}=\alpha_{4}=0$, and our main result for the expectation value of the current near the boundary is as follows:

$$
J_{b}=\frac{4 b_{1} F_{b n}}{x}, \quad x \sim 0,
$$

We emphasis that the current [Eq. (4)] does not involve any on-shell charged particle as we were considering the vacuum state and there is no Schwinger effect for the magnetic field. Instead the induced current should be identified with a magnetization current as a result of the magnetization [Eq. (5)] of the vacuum. This can be derived directly without first referring to the current [Eq. (4)] by using the magnetic coupling $S=\int_{M} \sqrt{g} \boldsymbol{M} \cdot \boldsymbol{B}$ and the relation

$$
(\delta \mathcal{A})_{\partial M_{\epsilon}}=\left(\int_{M} \sqrt{g} \boldsymbol{M} \cdot \delta \boldsymbol{B}\right)_{\log _{\frac{e}{e}} \frac{1}{2}}
$$

By considering a variation $\delta B_{z}=\delta(x-\epsilon) \delta f(y, z)$ that is localized on the boundary $\partial M$, one obtains Eq. (5).

The universal laws [Eqs. (4) and (5)] hold for general BQFTs, which are covariant, gauge invariant, unitary, and renormalizable, or, equivalently, for BQFTs whose Weyl anomaly is given by Eq. (3). Several comments are in order. (1) Since Eqs. (4) and (5) depend on only the bulk central charge instead of boundary central charge, it is independent of the choices of boundary conditions. Thus the current is more universal than the renormalized stress tensor near the boundary, which depends on boundary conditions $[17,33,38,39]$. (2) The magnitude of the induced current is much larger than that of the stress tensor. To see this, let us recover the units in the formula. We have 
$J_{b}=\left(e^{2} c / \hbar\right)\left(4 b_{1} F_{b n} / x\right), T_{a b}=\hbar c\left(d_{1} h_{a b} / x^{4}\right)$, where $e$ is the charge, $c$ is the speed of light, $\hbar$ is the Planck constant, $b_{1}, d_{1}$ are dimensionless constants, and $h_{a b}$ is the boundary metric. We have rescaled $F_{\mu \nu} \rightarrow e F_{\mu \nu}$ so that the field strength is related to electric field and magnetic field in the usual manner: $E_{i}=c F_{i 0}, B_{i}=\frac{1}{2} \epsilon_{i j k} F^{j k}$. (3) Our result shows that the constant magnetic field parallel to the boundary can induce a current [Eq. (4)]. As we illustrated above, the boundary is crucial in realizing a separation of charges that result in the induced anomalous current and in the nonuniform magnetization for the vacuum. (4) We emphasize that our current is not due to the on-shell movement of charges, but to the transport of virtual charges as a result of nonuniform vacuum magnetization. As such our current does not obey Ohm's law and is not dissipative. It does not require an energy source to support it. (5) The result [Eq. (4)] is for a single boundary. For a real system with finite extent, e.g., a rectangular slab with two parallel boundaries, we will have the current of the same form near each boundary component of the system. The total current is zero and satisfies the Bloch theorem [40]. (6) The relation [Eq. (9)] also implies an induced charge density $\rho=\left(e^{2} / \hbar\right)\left(4 b_{1} E / x\right)$ near the boundary. Here $\boldsymbol{E}=E \boldsymbol{e}_{x}$. (7) Our results [Eqs. (4) and (5)] were derived for the vacuum. In a material system, one needs to take into account of the presence of charge carriers and nonvanishing conductivity of the media. The direct field theory analysis seems rather complicated. However due to the close relation with the Weyl anomaly, we expect that these results will continue to hold. In Ref. [41] we use a holographic model to study the effect of conductivity, and we find that the current and the magnetization are not modified in the leading order of closeness to the boundary.

Story of free QFT.-Our general result [Eq. (9)] is verified by a free BQFT. For simplicity, let us consider complex scalar field with the action $I=-\int_{M} \sqrt{g}$ $\left[\left(D^{\mu} \phi\right)^{*} D_{\mu} \phi+E \phi^{*} \phi\right]$, where $D_{\mu}=\partial_{\mu}+i A_{\mu}$ are gauge invariant covariant derivatives and $E$ is a function that includes only the coupling constants with a non-negative mass dimension. In general, there are two kinds of boundary conditions for the scalar [42]: Dirichlet BC $\left(\left.\phi\right|_{\partial M}=0\right)$ and the Robin BC $\left[\left.\left(D_{n}+\psi\right) \phi\right|_{\partial M}=0\right]$. Here the function $\psi$ defines a renormalizable theory. For a free complex scalar field theory, the expectation value of the current near the boundary has been derived in Ref. [42] using heat kernel expansion. The result is $J_{b}=$ $-\left(F_{b n} / 24 \pi^{2} x\right)$ for both Dirichlet BC and Robin BC. The Weyl anomaly for the complex scalar theory can be derived as the heat-kernel coefficient $a_{4}$ [43,44]. In this way, we get the Weyl anomaly [Eq. (3)] with the central charge $b_{1}=-1 / 96 \pi^{2}$. It is clear that the obtained current indeed satisfies our derived universal law [Eq. (9)]. From this simple example, we have learned two important facts. First, the near-boundary current is indeed independent of the choices of boundary conditions. Second, the universal law
[Eq. (9)] works for not just BCFT, but also for more general QFT. The only constraints we impose on the functions $E, \psi$ are that they define a renormalizable theory. In particular, the theory need not be conformal invariant.

Finite total current.-Similar to the case of a stress tensor $[17,38,45]$, there are boundary contributions to the current that make the total current finite. To see this, consider the gauge variation of the finite part of the effective action. Due to gauge invariance, we obtain the conservation laws $D_{\mu} J^{\mu}=0$ in the bulk and $\mathcal{D}_{a} j^{a}=-J^{n}$ on the boundary. From the bulk current conservation and Eq. (9), we get $J_{n}=4 b_{1} \mathcal{D}_{b} F_{n}^{b} \ln x+O(1)$. Substituting $J_{n}$ into the boundary conservation law, we obtain the boundary current $j_{b}=4 b_{1} F_{b n} \ln \epsilon$. As a result, we have

$$
J_{b}=\frac{4 b_{1} F_{b n}}{x}+\delta(x ; \partial M) 4 b_{1} F_{b n} \ln \epsilon+O(1),
$$

where we have shifted the boundary from $x=0$ to a position $x=\epsilon$. It is remarkable that the boundary current obtained from the conservation law automatically yields the total current [Eq. (11)], which represents a finite flow of charge through any interval in the normal direction.

On experimental observation.-Our current [Eq. (4)] can be observed by measuring the magnetic response of the vacuum to the external field in the presence of boundary. We have shown that the renormalized current and the quantum magnetization are independent of the choices of well-defined boundary conditions (BC). By "well-defined $\mathrm{BC}$," we mean that no current can flow out of the boundary. The insensitivity of BCs would decrease the difficulty in experiments. In reality, since modes with sufficiently high frequencies would penetrate the boundary, this corresponds to an effective length cutoff and our formula [Eq. (9)] will work well only for $x>\epsilon$ with the cutoff naturally being the lattice length $a_{\text {lattice }}$ of the material in consideration. Consider, for simplicity, a constant magnetic field $B$ and constant temperature $T$ for the material. On the other hand, the formula [Eq. (9)] applies only to the region close enough to the boundary such that $x<x_{\max }=$ $\min \left(\hbar c /(k T), \hbar /\left(c m_{\text {eff }}\right), \sqrt{\hbar /(e B)}\right)$, where $m_{\text {eff }}$ is the effective mass of the charged particle. Taking $T=300 \mathrm{~K}$, $m_{\mathrm{eff}}=m_{e}$ to be the mass of electron and $B=0.01 \mathrm{~T}$, we have $x_{\max } \sim \min \left(10^{-5} \mathrm{~m}, 10^{-13} \mathrm{~m}, 10^{-6} \mathrm{~m}\right)$, which shows that the large mass of charged particle is the main obstruction to experimental observation of the phenomena. Thus one must try to decrease the effective mass in materials in order to satisfy $\epsilon<x_{\max }$. Fortunately, the availability of charge carriers with zero effective mass in graphene [46] and Dirac or Weyl semimetals [47] makes these systems a more promising setup for the experimental observation of this induced current phenomena.

Conclusions and discussions. - In this Letter, we show that, for general BQFTs that are gauge invariant, unitary, and renormalizable, the renormalized current takes the 
universal form [Eq. (9)] near the boundary. This covers fundamental theories such as QED, as well as many typical condensed matter systems of interests. The induced current is independent of the boundary conditions and the states of $\mathrm{BQFT}$, and depends only on the beta function of the theory. Since the current is proportional to the quantum Hall conductance $e^{2} / \hbar$, it is potentially large enough to be measured experimentally. It is interesting to perform an experiment to observe this effect. It is also interesting to look for a suitable implication of this effect for other physical systems such as astronomical objects or branes in string theory. Our discussions can be easily generalized to a system with a background non-Abelian gauge field and with spacetime dimensions other than four (see Supplemental Material [34]). We note however that only in four dimensions is the near boundary value of the current determined universally by the bulk central charge and is independent of boundary conditions.

We thank B. L Hu, Y. Liu, J.X. Lu, D. W. Wang, and L. Y Hung for useful discussions. This work is supported in part by NCTS and Grant No. MOST 105-2811-M-007-021 of the Ministry of Science and Technology of Taiwan. R. X. Miao thank the funding of Sun Yat-Sen University.

[1] For a review, see, for example, D. E. Kharzeev, Prog. Part. Nucl. Phys. 75, 133 (2014); K. Landsteiner, Acta Phys. Pol. B 47, 2617 (2016).

[2] A. Vilenkin, Astrophys. J. 451, 700 (1995).

[3] A. Vilenkin, Phys. Rev. D 22, 3080 (1980).

[4] M. Giovannini and M. E. Shaposhnikov, Phys. Rev. D 57, 2186 (1998).

[5] A. Y. Alekseev, V. V. Cheianov, and J. Frohlich, Phys. Rev. Lett. 81, 3503 (1998).

[6] K. Fukushima, Lect. Notes Phys. 871, 241 (2013).

[7] D. Kharzeev and A. Zhitnitsky, Nucl. Phys. A797, 67 (2007).

[8] J. Erdmenger, M. Haack, M. Kaminski, and A. Yarom, J. High Energy Phys. 01 (2009) 055.

[9] N. Banerjee, J. Bhattacharya, S. Bhattacharyya, S. Dutta, R. Loganayagam, and P. Surowka, J. High Energy Phys. 01 (2011) 094.

[10] D. T. Son and P. Surowka, Phys. Rev. Lett. 103, 191601 (2009).

[11] K. Landsteiner, E. Megias, and F. Pena-Benitez, Phys. Rev. Lett. 107, 021601 (2011).

[12] M. N. Chernodub, Phys. Rev. Lett. 117, 141601 (2016).

[13] M. N. Chernodub, A. Cortijo, and M. A. H. Vozmediano, Phys. Rev. Lett. 120, 206601 (2018).

[14] H. B. G. CasimirIndagat. Math 10, 261 (1948) [, Kon. Ned. Akad. Wetensch. Proc. 51, 793 (1948)] [Front. Phys. 65, 342 (1987)] [Kon. Ned. Akad. Wetensch. Proc. 100N3-4, 61 (1997)].

[15] G. Plunien, B. Muller, and W. Greiner, Phys. Rep. 134, 87 (1986).

[16] M. Bordag, U. Mohideen, and V. M. Mostepanenko, Phys. Rep. 353, 1 (2001).
[17] R. X. Miao and C. S. Chu, J. High Energy Phys. 03 (2018) 046.

[18] J. L. Cardy, arXiv:hep-th/0411189.

[19] For a review, see, for example, M. Z. Hasan and C. L. Kane, Rev. Mod. Phys. 82, 3045 (2010).

[20] M. J. Duff, Classical Quantum Gravity 11, 1387 (1994).

[21] L. S. Brown, Phys. Rev. D 15, 1469 (1977).

[22] S. Deser and A. Schwimmer, Phys. Lett. B 309, 279 (1993).

[23] For parity-odd theory, it is possible that the Weyl anomaly includes one additional term [24-26] $\Delta \mathcal{A}=b_{2} \int_{M} \sqrt{g} \epsilon^{i j k l}$ $F_{i j} F_{k l}$. This is just a total derivative term and gives the Chern-Simons action on the boundary after the integral. However, it is controversial whether such terms are allowed [27]. Even if such terms are allowed, [26] notice that the coefficients $b_{2}$ are imaginary, which would violate the unitarity. Thus, for the unitary QFT we are interested in, the most general form of Weyl anomaly is given by Eq. (3).

[24] M. J. Duff, Ultraviolet Divergences in Extended Supergravity, Supergravity 81, edited by S. Ferrara and J. G. Taylor (Cambridge University Press, Cambridge, England, 1982).

[25] Y. Nakayama, Nucl. Phys. B859, 288 (2012).

[26] L. Bonora, S. Giaccari, and B. Lima de Souza, J. High Energy Phys. 07 (2014) 117.

[27] F. Bastianelli and R. Martelli, J. High Energy Phys. 11 (2016) 178.

[28] D. Fursaev, J. High Energy Phys. 12 (2015) 112.

[29] C. P. Herzog, K. W. Huang, and K. Jensen, J. High Energy Phys. 01 (2016) 162.

[30] M.E. Peskin and D. V. Schroeder, An Introduction to Quantum Field Theory (Addison-Wesley, Reading, 1995).

[31] Below, we will spell out explicitly up to what distance scale the result holds.

[32] One should not mix this up with the quantum anomalous Hall effect, which refers to a quantized Hall effect realized in the system without an external magnetic field.

[33] D. Deutsch and P. Candelas, Phys. Rev. D 20, 3063 (1979).

[34] See Supplemental Material at http://link.aps.org/ supplemental/10.1103/PhysRevLett.121.251602 for details on relation between Weyl anomaly and current, which includes Refs. [35-37].

[35] A. Lewkowycz and E. Perlmutter, J. High Energy Phys. 01 (2015) 080.

[36] X. Dong, Phys. Rev. Lett. 116, 251602 (2016).

[37] J. S. Dowker and J. P. Schofield, J. Math. Phys. (N.Y.) 31, 808 (1990).

[38] G. Kennedy, R. Critchley, and J. S. Dowker, Ann. Phys. (N.Y.) 125, 346 (1980).

[39] G. Kennedy, Ann. Phys. (N.Y.) 138, 353 (1982).

[40] N. Yamamoto, Phys. Rev. D 92, 085011 (2015).

[41] C. S. Chu, R. X. Miao et al. (to be published).

[42] D. M. McAvity and H. Osborn, Classical Quantum Gravity 8, 603 (1991).

[43] D. V. Vassilevich, Phys. Rep. 388, 279 (2003).

[44] We thank Duff for the private communication.

[45] J. S. Dowker and G. Kennedy, J. Phys. A 11, 895 (1978). 
[46] M. A. H. Vozmediano, M. I. Katsnelson, and F. Guinea, Phys. Rep. 496, 109 (2010); F. de Juan, M. Sturla, and M. A. H. Vozmediano, Phys. Rev. Lett. 108, 227205 (2012); J. Scott Bunch, S. S. Verbridge, J. S. Alden, A. M. van der Zande, J. M. Parpia, H. G. Craighead, and P. L. McEuen, Nano Lett. 8, 2458 (2008); G. E. Volovik and M. A. Zubkov, Ann. Phys. (Amsterdam) 340, 352 (2014).
[47] O. Parrikar, T. L. Hughes, and R. G. Leigh, Phys. Rev. D 90, 105004 (2014); M. A. Zubkov, Ann. Phys. (Amsterdam) 360, 655 (2015); A. Cortijo, Y. Ferreiros, K. Landsteiner, and M. A. H. Vozmediano, Phys. Rev. Lett. 115, 177202 (2015); A. Cortijo and M. A. Zubkov, Ann. Phys. (Amsterdam) 366, 45 (2016). 